\title{
Muography applied to nuclear waste storage sites
}

\author{
Raffaello D’Alessandro ${ }^{1,2,{ }^{\star}, \text { Fabio Ambrosino }}{ }^{3,4}$, Lorenzo Bonechi ${ }^{2}$, Luigi Cimmino ${ }^{1,2}$, \\ David G. Ireland ${ }^{5}$, Ralf Kaiser ${ }^{5}$, David F. Mahon ${ }^{5}$, Nicola Mori ${ }^{1,2}$, Pasquale Noli ${ }^{4}$, \\ Giulio Saracino $^{3,4}$, Craig Shearer ${ }^{6}$, Lorenzo Viliani ${ }^{1,2}$, Guangliang Yang ${ }^{5}$
}

\author{
${ }^{1}$ Università di Firenze, Dipartimento di Fisica e Astronomia, Sesto Fiorentino, Florence, Italy \\ ${ }^{2}$ Istituto Nazionale di Fisica Nucleare (INFN), Sezione di Firenze, Sesto Fiorentino, Florence, Italy \\ ${ }^{3}$ Università di Napoli Federico II, Dipartimento di Fisica, Naples, Italy \\ ${ }^{4}$ Istituto Nazionale di Fisica Nucleare (INFN), Sezione di Napoli, Naples, Italy \\ 5 SUPA, School of Physics and Astronomy, University of Glasgow, Glasgow, Scotland, UK \\ ${ }^{6}$ National Nuclear Laboratory, Central Laboratory, Sellafield, Seascale, Cumbria, England, UK
}

\author{
Article history \\ Received July 5, 2016; accepted October 5, 2016. \\ Subject classification: \\ Muons, Radiography, Nuclear waste.
}

\begin{abstract}
Legacy storage sites for nuclear waste can pose a serious environmental problem. In fact, since certain sites date from the middle of the last century when safety protocols had not been properly established and strict bookkeeping was not enforced, a situation has evolved where the content of storage silos is basically known only with a large uncertainty both on quantity and quality. At the same time maintenance work on old storage structures is becoming ever more urgent and yet this work requires exactly that information which is now lacking on the type of waste that was stored inside. Because of the difficulty in accessing the storage silos and the near impossibility of making visual inspections inside, techniques have to be developed which can determine the presence or absence of heavy elements (i.e. uranium) within the structures. Muography is a very promising technique which could allow the survey of previously inaccessible structures. We have begun an evaluation performing feasibility studies using simulations based on real case scenarios. This paper will outline the storage site scenarios and then present some of the results obtained from the Monte Carlo simulations.
\end{abstract}

\section{Introduction}

The monitoring of legacy storage sites for nuclear waste is growing the interest of the nuclear industry. The limited knowledge of the content of some storage facilities coming from an epoch when there were no strict rules for bookkeeping impairs the ability to perform the needed maintenance operations. Investigation campaigns on these sites are strongly constrained by factors like the difficult access to high-radiation environments and the impossibility to make visual inspections. Given the critical importance of this topic in the frame of environment conservation, new inspection methods which cope with these difficulties must be developed.

Muon radiography is a technique that is attracting much attention in many fields related to the investigation of the internal density structure of a given target body. Its application to the detection and monitoring of high- $Z$ materials (such as uranium) inside various kind of containers ranging from a nuclear reactor [Morris et al. 2014, Miyadera et al. 2013] to cargos [Blackwell and Kudryavtsev 2013, Riggi et al. 2013] has been studied recently. Monte Carlo simulations also show that muon radiography can be successful in detecting $\mathrm{cm}$-sized uranium samples inside small storage canisters filled with concrete [Clarkson et al. 2015]. This result, however, does not provide any firm evidence that the technique can scale up to storage facilities with typical dimensions of several meters.

When enlarging the size of the inspected structure, two kinds of problems arise. The first one is connected to the geometric acceptance of the detection device and thus to the data acquisition time needed to collect a reasonable amount of muons. Typically, the presence of high- $Z$ materials is investigated by measuring the scattering position inside the target and the scattering angle of an incoming muon: a large number of muons will be scattered at large angles when traversing dense regions inside the inspected structure. This technique requires at least two tracking devices, one upstream of the structure, to measure the direc- 
tion of the incoming muon, and one downstream, to measure the direction after traversing the structure. The distance between the trackers when they are placed at opposite sides of a large storing facility will reduce the geometric acceptance of the detection device, making the inspection of large volumes unfeasible (although the monitoring of a localized sample with known position would still be possible by means of a proper arrangement of the detectors). The second problem with large facilities is the increased background due to multiple scattering inside the structure itself. A shield of concrete which is several meters thick can add a non-negligible contribution to the scattering angle given by the passage through the high- $Z$ region, and increase the probability of a large-angle scattering in the concrete itself. These effects must be assessed by means of dedicated studies.

In this paper we present the preliminary results of a Monte Carlo study of the performance of a muon radiography technique based on muon absorption when applied to large storage volumes. Muon absorption is widely used in investigating large geological structures [Tanaka et al. 2001, Ambrosi et al. 2011, Cârloganu et al. 2013] thanks to its large acceptance given by the usage of a single, downstream detector. In the next sections we describe the measurement scenario (storage silo, detector, measurement principle) and the data analysis techniques and then we briefly discuss the results.

\section{Measurement scenario}

In order to obtain results which are relevant to the real world, a real storage scenario has been considered. The storage facility is a cylindrical silo filled with concrete, with radius $3.5 \mathrm{~m}$ and height $4 \mathrm{~m}$ : this kind of facility is present e.g. in the storage site managed by Sellafield Ltd. (UK). The hypothesized uranium content of the real silos is an unknown number of $\mathrm{cm}$-sized uranium debris in unknown locations.

A Monte Carlo simulation based on Geant4 [Agostinelli et al. 2003] for the above described scenario has been set up. The simulated silo contains various uranium cubes with sizes of 2,5 and $10 \mathrm{~cm}$ at different locations, and a cubic air bubble with a size of $10 \mathrm{~cm}$ in order to assess the effects of eventual low-density regions inside the concrete. The detector is made of two parallel planes spaced by $50 \mathrm{~cm}$; each plane is $(2 \times 2) \mathrm{m}^{2}$ wide and consists of two orthogonal layers of scintillating bars made of poly-vinyl-toluene. The assumed spatial resolution of the detector is $0.3 \mathrm{~cm}$. These figures have been chosen in order to resemble a realistic, scaledup muon detector for geological surveys [Ambrosi et al. 2011]. The simulation setup is shown in Figure 1.

A total number of $1.2 \times 10^{9}$ events inside the de-

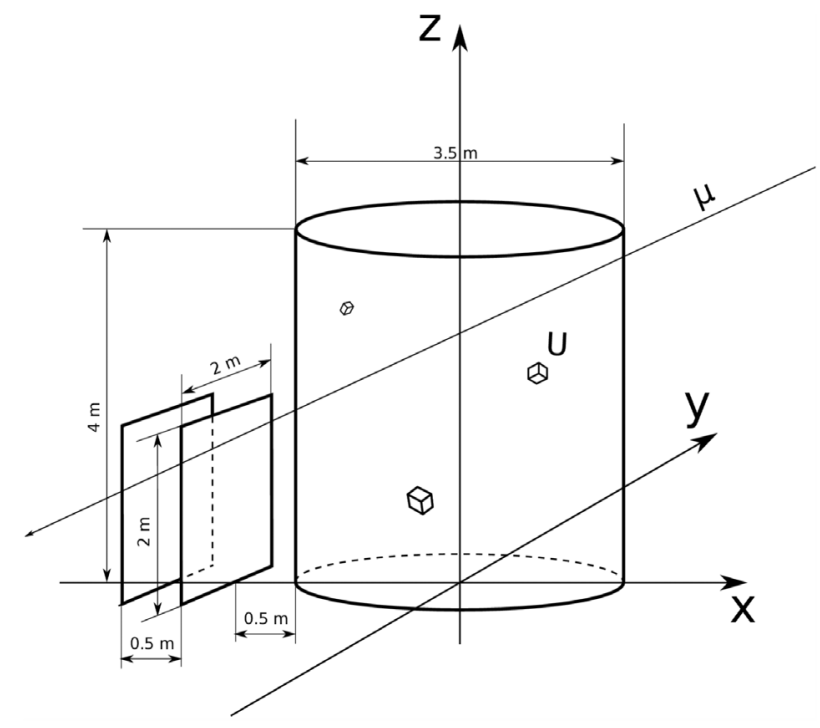

Figure 1. Schematic representation of the simulated scenario. The direction of arrival of detected muons is from right to left.

tector acceptance have been simulated, which corresponds to a data acquisition time of about 160 days. The energy and angular spectra of the muon flux used for random particle generation have been parameterized from ground measurements performed with a magnetic spectrometer [Bonechi et al. 2005]. The simulated energy range is $0.7-130 \mathrm{GeV}$, while the polar angle ranges from 0 (i.e. vertical muon) to 80 degrees.

\section{Data analysis}

The absorption technique seeks for missing muons due to enhanced absorption when traversing high-density regions inside the target. This requires an estimate of the expected number of detected events coming from any direction when no density fluctuation is present, in order to compute the amount of missing events from a given direction which will be proportional to the column density along that direction. For large targets like geological structures this method can only provide information about the column density of the target along a line of sight, since the detector is point-like when compared to the dimension of the target, thus resulting in a two-dimensional density map. However, in the storage silo scenario the dimension of the detector is not negligible, and this fact can be exploited to obtain a full three-dimensional estimate of the target density. As shown in Figure 2, missing muon tracks due to a localized high-density region inside the target are detected along different directions, allowing for a stereoscopic reconstruction of the position of the absorption point. Detected tracks can be back-extrapolated to the silo region, the intersections with $Y Z$ planes at a given $X$ can be computed and from those a YZ histogram of intersection points can be built. This histogram (the "back projection map") will show a deficit of events with respect to the 


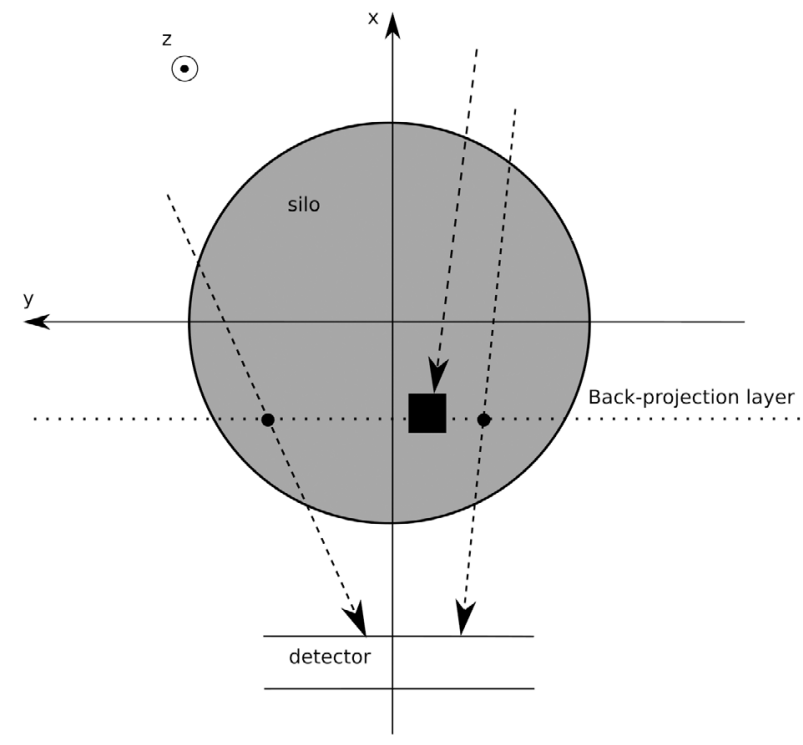

Figure 2. Measurement principle: missing tracks (dashed lines) due to absorption in a high-density region (black box) "hit" the detector with different angles, allowing for a stereoscopic view of the absorption point.

homogeneous target case in the portion of the histogram (the "signal region") where the YZ plane intersects the high-density region inside the silo. Together with the $\mathrm{X}$ position of the back-projection plane, the $\mathrm{YZ}$ position of the deficit will give the full three-dimensional location of the high-density region. If the plane does not intersect the high density region then the signal region will be wider and the signal-to-noise will be less, analogously to what happens to optical images outside the focal plane of the optical system. Many back-projection layers at different $\mathrm{X}$ coordinates can thus be considered, and the $\mathrm{X}$ position of the high-density region will be that of the layer giving the smallest signal region with the highest signal-to-noise ratio. This method is described in details in Bonechi et al. [2015].

The above described method requires the knowledge of the expected event count for a silo containing no uranium debris. This information cannot be measured directly since the content of the silos is unknown and thus there is no known uranium-free silo. However, since uranium debris are much smaller than the silo and their positions are scattered randomly in the silo volume, it seems reasonable to assume that the position of a debris inside a silo will not match any of the positions of the debris inside another silo, so that the signal regions of the back projection maps at a given $\mathrm{X}$ for the two silos will never overlap. With this assumption, subtracting the back projection map of silo A from that of silo $\mathrm{B}$ will show signal regions for $\mathrm{B}$ as regions with a deficit of events (direct signal), and those for $A$ as an excess of events (inverse signal). In order to avoid the necessity of acquiring data for two silos, the mirror symmetry about the $Z$ axis of the measurement sce- nario can be exploited. Assuming that no uranium debris will lie exactly on the $Z$ axis, one can mirror the back projection map about the $Z$ axis and subtract it from the direct map. In this way, every direct signal region at coordinate $(\mathrm{Y}, \mathrm{Z})$ will have a mirrored inverse signal region at $(-Y, Z)$.

To investigate the presence of a signal from uranium debris and study its variation with acquisition time, simulated data have been analyzed by placing back projection layers so that they intersect the debris inside the silo. In a realistic scenario this would not be possible, since the positions of the debris would be unknown. A full stack of planes with a suitable regular spacing along $\mathrm{X}$ would be required to perform a tomographic scan of the silo, and suitable algorithms to find the plane containing the signal region with minimum extension and maximum signal-to-noise would have to be implemented in order to determine the position of the debris. This is far beyond the scope of this work, which only aims at assessing the eventual presence of a signal and its time dependence. So for this goal we relied on the knowledge of the positions given by the Monte Carlo truth. The simulated data have been processed with the mirror map technique in order to produce event count difference maps. Each bin of the difference maps is computed as the difference of two independent Poisson random variables (i.e. event counts), and thus follows a Skellam distribution whose RMS is given by the square root of the sum of the expectation values of the two Poisson variables, which have been estimated with the event count themselves. Signal-to-noise maps have thus been built by dividing each bin of the difference maps by its Skellam RMS.

An example of these maps is shown in Figure 3. The back projection layer is set at $\mathrm{X}=-100 \mathrm{~cm}$, where

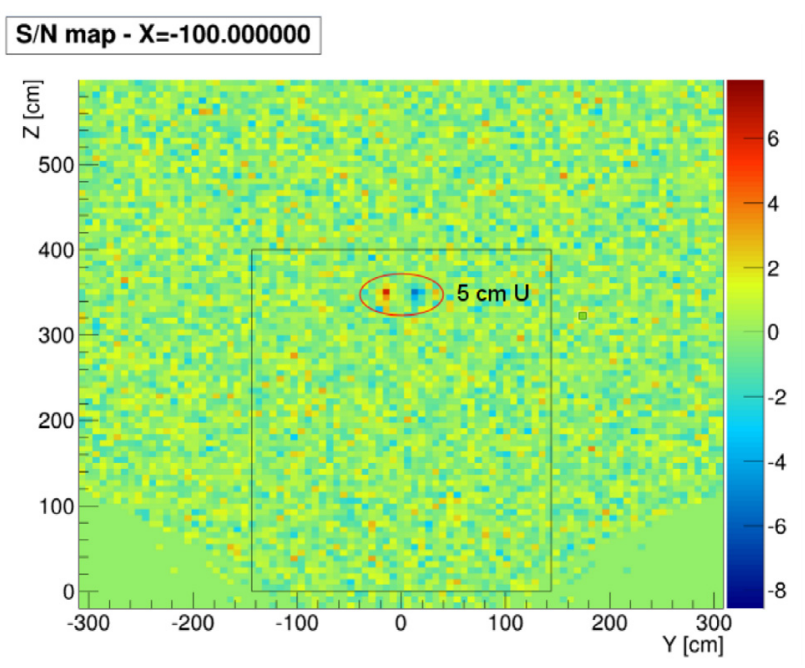

Figure 3. Signal-to-noise map for an acquisition time of about 160 days computed at $X=-100 \mathrm{~cm}$. The direct (red) and inverse (blue) signal regions are clearly visible at $Z \sim 350 \mathrm{~cm}$. 
a $5 \mathrm{~cm}$ uranium cube is present at coordinates $(\mathrm{Y}, \mathrm{Z})=$ $(-15,350) \mathrm{cm}$ (see Figures 1 and 2 for the details of the reference frame). The signal is clearly visible with a signal-to-noise value for the single bin around 6 , thanks to the uranium sample being placed near to the detector and in the upper part of the silo. This condition increase the statistics since the muon flux increase with decreasing polar angle, and makes the effect of multiple scattering in the concrete less effective due to the reduced thickness of traversed material. Indeed, the same $5 \mathrm{~cm}$ sample placed near the center of the silo does not produce a detectable signal. On the contrary, a $10 \mathrm{~cm}$ sample in the same position produce a signalto-noise of about 7 . The detection capability is thus strongly influenced by the position of the uranium debris, other than their size. To put a lower limit on this last parameter, $2 \mathrm{~cm}$ samples have been placed in various positions, but none of them resulted to give a detectable signal with the available statistics. Also, the 10 $\mathrm{cm}$ air bubble placed near the center of the silo resulted to be not visible as well.

In order to define the lower signal-to-noise threshold for detection, the probability of having a statistical fluctuation in a single bin giving a signal-to-noise greater than a given value has been studied. When taking into account the look-elsewhere effect given by the $\sim 2000$ bins corresponding to the silo region in the signal-to-noise map, it turns out that the probability of having one or more bins with a signal-to-noise ratio greater than 4 due to statistical fluctuations is about $9 \%$, decreasing to $0.08 \%$ when considering a threshold of 5. A signal-to-noise value of 5 have thus been assumed as a safe detection threshold. To assess the acquisition time needed to reliably detect a uranium sample, the analysis has been repeated by considering subsets of the whole simulation data set. A plot showing the dependence of the signal-to-noise ratio on the acquisition time for the $5 \mathrm{~cm}$ and the $10 \mathrm{~cm}$ visible samples is shown in Figure 4. An acquisition time of 1-2 months result to be sufficient to detect both the $5 \mathrm{~cm}$ and the $10 \mathrm{~cm}$ samples.

\section{Discussion}

In this work the feasibility of searching legacy nuclear waste storage silos for uranium presence leveraging the absorption of atmospheric muons has been investigated. This is a novel application of a well established and developed technique in the field of the monitoring of geological structures, which present many peculiar aspects when applied to the nuclear storage scenario. This preliminary analysis based on Monte Carlo simulations shows that in a realistic case there is a good discovery potential for well-positioned uranium

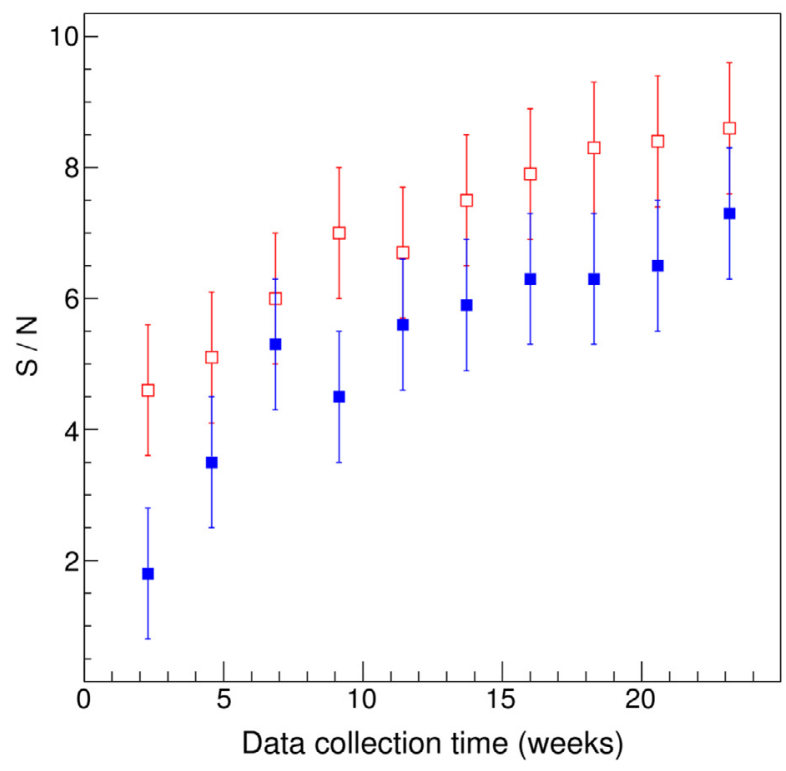

Figure 4. Signal-to-noise ratios for the $10 \mathrm{~cm}$ (open squares) and the $5 \mathrm{~cm}$ (full squares) samples as functions of the data acquisition time. Error bars represent the statistical errors.

debris with a typical size greater than $5 \mathrm{~cm}$. The required data acquisition time is of the order of a couple of months. The study of systematic effects given by e.g. non-uniform concrete density, the presence of other heavy debris like steel rods, surrounding buildings, different parameterizations of the muon flux etc. are beyond the scope of this preliminary, and will be addressed in a future work.

\section{References}

Agostinelli, S., al. (2003). Geant4 - a simulation toolkit, Nucl. Instrum. Meth. A, 506, 250-303.

Ambrosi, G., et al. (2011). The MU-RAY project: Volcano radiography with cosmic-ray muons, Nucl. Instrum. Meth. A, 628, 120-123.

Blackwell, T.B., and V.A. Kudryavtsev (2013). Identification of Nuclear Materials in Cargo Containers Using Cosmic Rays, Nuclear Science Symposium and Medical Imaging Conference (NSS / MIC), 1-8.

Bonechi, L., M. Bongi, D. Fedele, M. Grandi, S. Ricciarini and E. Vannuccini (2005). Development of the ADAMO detector: test with cosmic rays at different zenith angles, Proceedings of the 29th International Cosmic Ray Conference, 9, 283-286.

Bonechi, L., R. D’Alessandro, N. Mori and L. Viliani (2015). A projective reconstruction method of underground or hidden structures using atmospheric muon absorption data, J. Instrum., 10, P02003.

Cârloganu, C., et al. (2013). Towards a muon radiography of the Puy de Dôme, Geosci. Instrum. Meth., 2 (1), 55-60.

Clarkson, A., D.J. Hamilton, M. Hoek, D.G. Ireland, J.R. Johnstone, R. Kaiser, T. Keri, S. Lumsden, D.F. Mahon, 
B. McKinnon, M. Murray, S. Nutbeam-Tuffs, C. Shearer, G. Yang and C. Zimmerman (2015). Characterising encapsulated nuclear waste using cosmicray muon tomography, J. Instrum., 10, P03020.

Miyadera, H., K.N. Borozdin, S.J. Greene, E.C. Milner, C.L. Morris, Z. Luki, K. Masuda and J.O. Perry (2013). Imaging Fukushima Daiichi reactors with muons, AIP Advances, 3, 052133.

Morris, C.L., et al. (2014). Analysis of muon radiography of the Toshiba nuclear critical assembly reactor, Appl. Phys. Lett., 104, 024110.

Riggi, S., V. Antonuccio-Delogu, M. Bandieramonte, U. Becciani, A. Costa, P. La Rocca, P. Massimino, C. Petta, C. Pistagna, F. Riggi, E. Sciacca and F. Vitello (2013). Muon tomography imaging algorithms for nuclear threat detection inside large volume containers with the Muon Portal detector, Nucl. Instrum. Meth. A, 728, 59-68.

Tanaka, H., K. Nagamine, N. Kawamura, S.N. Nakamura, K. Ishida and K. Shimomura (2001). Development of the Cosmic-Ray Muon Detection System for Probing Internal-Structure of a Volcano, Hyperf. Int., 138 (1), 521-526.

*Corresponding author: Raffaello D’Alessandro, Istituto Nazionale di Fisica Nucleare (INFN), Sezione di Firenze, Sesto Fiorentino, Florence, Italy; email: candi@fi.infn.it.

(C) 2017 by the Istituto Nazionale di Geofisica e Vulcanologia. All rights reserved. 